\title{
Enantioselective Total Synthesis of Phomallenic Acid C
}

\author{
Ya-Jun Jian, Chao-Jun Tang and Yikang Wu* \\ State Key Laboratory of Bioorganic And Natural Products Chemistry, \\ Shanghai Institute of Organic Chemistry, Chinese Academy of Sciences, \\ 354 Fenglin Road, Shanghai 200032, China \\ yikangwu@.sioc.ac.cn
}

\section{Table of Contents}

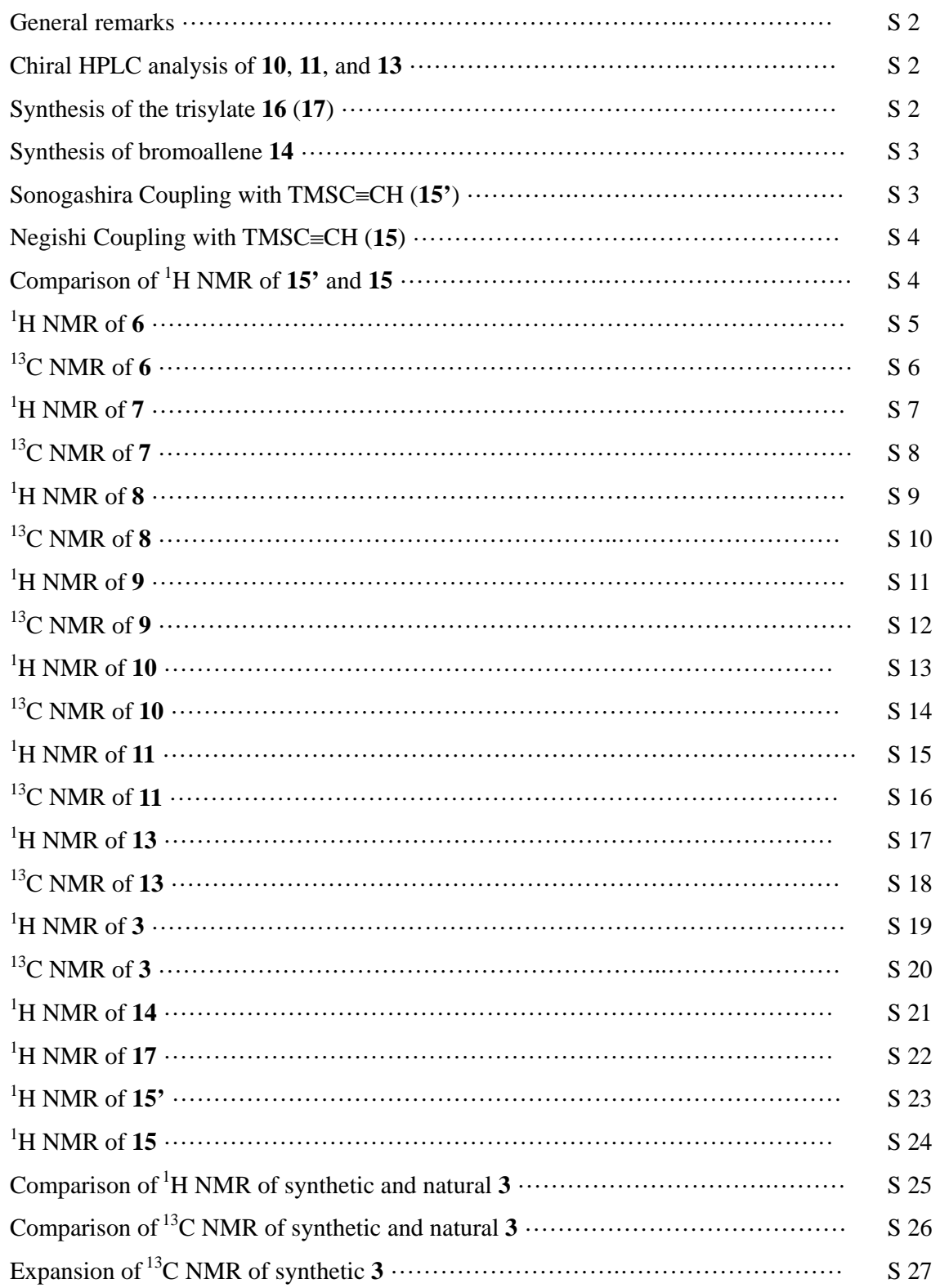




\section{General Remarks.}

Dry THF was distilled over $\mathrm{Na} / \mathrm{Ph}_{2} \mathrm{CO}$ under argon prior to use. Dry $\mathrm{CH}_{2} \mathrm{Cl}_{2}$ was distilled over $\mathrm{CaH}_{2}$ under argon prior to use. Column chromatography was performed on silica gel (300-400 mesh) under slightly positive pressure. PE stands for petroleum ether (bp $60-90^{\circ} \mathrm{C}$ ). The $\mathrm{LiBr}$ and $\mathrm{ZnBr}_{2}$ were dried in a flask by flame-dried under oil pump vacuum, respectively. ${ }^{1} \mathrm{H}$ NMR spectra were recorded at 300 or $400 \mathrm{MHz}$ in $\mathrm{CDCl}_{3}$ with reference to $\mathrm{Me}_{4} \mathrm{Si}$ unless otherwise indicated. ${ }^{13} \mathrm{C}$ NMR spectra were recorded at 75 or $100 \mathrm{MHz}$ in $\mathrm{CDCl}_{3}$ with reference to $\mathrm{Me}_{4} \mathrm{Si}$ unless otherwise indicated. IR spectra were recorded on an FT instrument with the oily sample smeared onto a $\mathrm{KBr}$ disc. EI-MS EI-HRMS, ESI-MS, ESI-HRMS data were all taken on commercially available instruments using direct injection technique. Optical rotations were measured at the sodium D line $(589 \mathrm{~nm})$ and are in units of $\mathrm{deg} \cdot \mathrm{mL}(\mathrm{dm} \cdot \mathrm{g})^{-1}$ with the concentration and solvent individually specified.

Chiral HPLC analysis. Separation of the enantiomers of tosylate $\mathbf{1 0}$ was carried out on a CHIRAL OD column of $0.46 \mathrm{~cm}$ (i.d.) $\times 25 \mathrm{~cm}$ (length), eluting with 100:1 $n$-hexane/2-propanol at a flow rate of $0.6 \mathrm{~mL} / \mathrm{min}$ with UV detection at $214 \mathrm{~nm}$. The retention times for the major $(R)$ and minor $(S)$-isomers were 88.6 and $83.9 \mathrm{~min}$, respectively, with the ee value $>96 \%$ (ratio $(R)-/(S)->49: 1$ ).

Separation of the enantiomers of bromoallene $\mathbf{1 1}$ was performed on a CHIRAPAK IC column of $0.46 \mathrm{~cm}$ (i.d.) $\times 25$ cm (length), eluting with 99:1 $n$-hexane/2-propanol at a rate of $1.0 \mathrm{~mL} / \mathrm{min}$ with UV detection at $220 \mathrm{~nm}$. The retention times for the major $(S)$ - and minor $(R)$-isomers were 41.9 and $38.7 \mathrm{~min}$, respectively, with the ee value $=90 \%($ ratio $(S)-/(R)-=20: 1)$.

Separation of the enantiomers of MOM ester 13 was performed on a CHIRAPAK IC column of $0.46 \mathrm{~cm}$ (i.d.) $\times 25$ $\mathrm{cm}$ (length), eluting with 99:1 $n$-hexane/2-propanol at a rate of $1.0 \mathrm{~mL} / \mathrm{min}$ with UV detection at $220 \mathrm{~nm}$. The retention times for the major $(R)$ - and minor $(S)$ - isomers were 54.0 and $73.7 \mathrm{~min}$, respectively, with the ee value $=88 \%(\operatorname{ratio}(R)-/(S)-=16: 1)$.

\section{Scheme S}

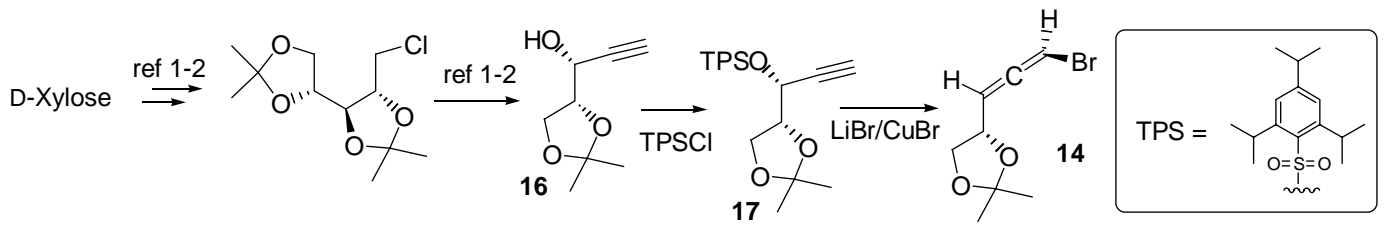

The bromoallene 14 in the main text was prepared (Scheme S) from the known alcohol 16 (ref.: (a) Kagawa, N.; Ihara, M.; Toyota, M. Org. Lett. 2006, 8, 875-878. (b) Mulzer, J.; Greifenberg, S.; Beckstett, A.; Gottwald, M. Liebigs. Ann. Chem. 1992, 1131-1135. (c) Yadav, J. S.; Chander, M. V.; Rao, C. S. Tetrahedron Lett. 1989, 30, 5455-5458.), which could be readily synthesized from D-xylose following the literature procedure (ref.: Yadav, J. S.; Chander, M. V.; Rao, C. S. Tetrahedron Lett. 1989, 30, 5455-5458.). Later, it was found that using $p$-TsCl to replace the TPSCl could also eventually 14 of the same quality.

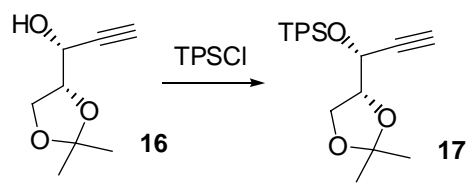

Trisylation of alcohol 16 (17). 2,4,6-Triisopropylbenzenesulfonyl chloride (2.160 g, 7.15 mmol) was added in portions to a solution of the alcohol 16 (1.105 g, $7.08 \mathrm{mmol})$ and DMAP (1.045 g, $8.56 \mathrm{mmol})$ in $\operatorname{dry~}_{\mathrm{CH}} \mathrm{Cl}_{2}(22 \mathrm{~mL})$ 
stirred at ambient temperature. After completion of the addition, the mixture was heated to $40^{\circ} \mathrm{C}$ (bath) with stirring until TLC showed completion of the reaction. Sat. aq. $\mathrm{NH}_{4} \mathrm{Cl}$ and EtOAc were added to the reaction mixture. The phases were separated. The organic layer was washed with brine and dried over anhydrous $\mathrm{Na}_{2} \mathrm{SO}_{4}$. Removal of the solvent by rotary evaporation and chromatography on silica gel (1:10 EtOAc/PE) gave the trisylate $\mathbf{1 7}$ as a colorless oil (2.670 g, $6.33 \mathrm{mmol}, 89 \%$ yield): $[\alpha]_{\mathrm{D}}{ }^{25}-8.9$ (c 1.80, $\left.\mathrm{CHCl}_{3}\right) .{ }^{1} \mathrm{H}$ NMR (300 MHz, $\left.\mathrm{CDCl}_{3}\right) \delta 7.17$ (s, $\left.2 \mathrm{H}\right), 5.16$ (dd, $J=6.9,2.5 \mathrm{~Hz}, 1 \mathrm{H}$ ), 4.34 (qd, $J=6.4,1.7 \mathrm{~Hz}, 1 \mathrm{H}$ ), 4.19-4.10 (m, 3H), 4.05 (dd, $J=9.1,5.4 \mathrm{~Hz}, 1 \mathrm{H}$ ), 2.92 (heptet, $J=$ $7.0 \mathrm{~Hz}, 1 \mathrm{H}), 2.37$ (d, $J=2.2 \mathrm{~Hz}, 1 \mathrm{H}), 1.37$ (s, 3H), 1.34 (s, 3H), 1.30-1.25 (m, 18H); FT-IR (film) 3278, 2131, 1598, $1178 \mathrm{~cm}^{-1}$; EI-MS m/z (\%) 407 (M-CH3, 35.4), 283 (61.7), 218 (53.3); 101 (100); 43 (47.7); HRMS calcd for $\mathrm{C}_{22} \mathrm{H}_{31} \mathrm{O}_{5} \mathrm{~S}\left(\left[\mathrm{M}-\mathrm{CH}_{3}\right]^{+}\right)$407.1892; found 407.1880.

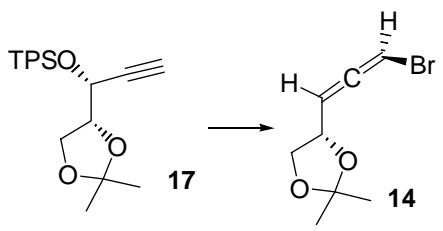

Conversion of 17 into bromoallene 14. A solution of the sulfonate $(780 \mathrm{mg}, 1.85 \mathrm{mmol})$ in dry THF ( $4.5 \mathrm{~mL}$, another $3 \mathrm{~mL}$ to assist the transfer) was added via a syringe to a dry flask containing $\mathrm{CuBr} \cdot \mathrm{SMe}_{2}(1.410 \mathrm{~g}, 5.55 \mathrm{mmol}$, recrystallized) and $\mathrm{LiBr}$ (481 mg, $5.55 \mathrm{mmol}$, dried) stirred at ambient temperature under argon. The mixture was heated to reflux under argon for $3 \mathrm{~h}$. After being cooled to ambient temperature, the reaction mixture was diluted with EtOAc, washed with sat. aq. $\mathrm{NH}_{4} \mathrm{Cl}$, and dried over anhydrous $\mathrm{Na}_{2} \mathrm{SO}_{4}$. Removal of the solvent and chromatography (1:3 EtOAc/PE) gave bromoallene 14 as a colorless oil (357 mg, $1.63 \mathrm{mmol}, 88 \%$ yield): $[\alpha]_{\mathrm{D}}{ }^{18}-84.4$ (c 1.58, $\mathrm{CHCl}_{3}$ ). ${ }^{1} \mathrm{H}$ NMR (300 MHz, CDCl $) \delta 6.12$ (dd, $J=5.9,1.1 \mathrm{~Hz}, 1 \mathrm{H}$ ), 5.43 (dd, $J=7.1,6.3 \mathrm{~Hz}, 1 \mathrm{H}$ ), 4.69 (qd, $J=6.4,1.2 \mathrm{~Hz}$, 1H), 4.20 (dd, $J=8.1,5.9 \mathrm{~Hz}, 1 \mathrm{H}$ ), 3.81 (dd, $J=8.2,6.1 \mathrm{~Hz}, 1 \mathrm{H}$ ), 1.44 (s, 3H), 1.39 (s, 3H); FT-IR (film) 2987, 1961, $1220 \mathrm{~cm}^{-1}$; EI-MS m/z (\%) $220\left(\mathrm{M}+\mathrm{H}^{+}\right.$, 3), 101 (100), 43 (88). Anal. Calcd. for $\mathrm{C}_{8} \mathrm{H}_{11} \mathrm{BrO}_{2}$ : C 43.86, H 5.06. Found: C 43.84, H 5.40 .

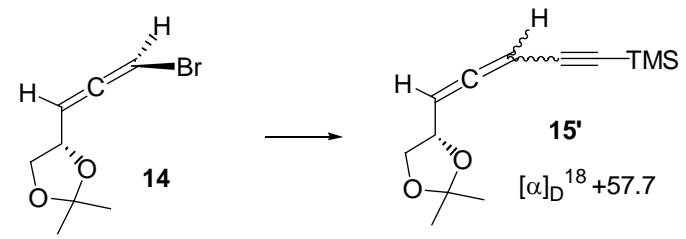

Coupling of 14 with TMSC $\equiv \mathbf{C H}$ under the Sonogashira conditions (15'). $\mathrm{PdCl}_{2}\left(\mathrm{PPh}_{3}\right)_{2}(19.3 \mathrm{mg}, 27.5 \mu \mathrm{mol}), \mathrm{CuI}$ (17.2 $\mathrm{mg}, 90 \mu \mathrm{mol})$, and $i-\mathrm{Pr}_{2} \mathrm{NH}(1.7 \mathrm{~mL}$ ) were quickly added in turn to a solution of the bromoallene (80 $\mathrm{mg}, 0.36$ $\mathrm{mmol})$ and $\mathrm{TMSC} \equiv \mathrm{CH}(70 \mathrm{mg}, 0.57 \mathrm{mmol})$ in dry de-aired EtOAc $(13 \mathrm{~mL})$ at $-20^{\circ} \mathrm{C}$ under argon. The mixture was stirred while the temperature was allowed to rise to $0^{\circ} \mathrm{C}$ over $30 \mathrm{~min}$. The stirring was then continued at ambient temperature until TLC showed completion of the reaction. Sat. aq. $\mathrm{NH}_{4} \mathrm{Cl}$ and $\mathrm{Et}_{2} \mathrm{O}$ were added. The phases were separated. The organic layer was washed with brine and dried over anhydrous $\mathrm{Na}_{2} \mathrm{SO}_{4}$. Rotary evaporation and column chromatography on silica gel (1:30 $\left.\mathrm{Et}_{2} \mathrm{O} / \mathrm{PE}\right)$ gave the allen-yne $\mathbf{1 5}^{\prime}$ as a yellowish oil $(65 \mathrm{mg}, 0.28 \mathrm{mmol}$, 77\%): $[\alpha]_{\mathrm{D}}{ }^{18}+57.7$ (c 0.65, $\left.\mathrm{CHCl}_{3}\right) .{ }^{1} \mathrm{H}$ NMR $\left(300 \mathrm{MHz}, \mathrm{CDCl}_{3}\right) \delta$ 5.58-5.49 (m, 2H), 4.67-4.62 (m, 1H), 4.21-4.12 (m, 1H), 3.81-3.74 (m, 1H), 1.45 (s, 3H), 1.40 (s, 3H), 0.18 (s, 9H). Cf. the attached spectrum (Figure 1). 


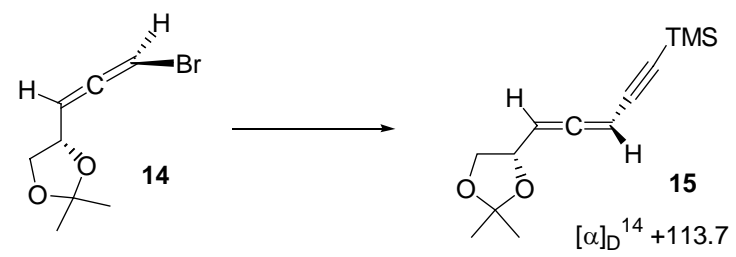

Coupling of $\mathbf{1 4}$ with TMSC $\equiv \mathbf{C H}$ under the Negishi conditions (15). $n$-BuLi $(0.80 \mathrm{~mL}$, ca $1.6 \mathrm{M}$ in hexanes, 1.28 mmol) was added via a syringe to a solution of the TMSC $\equiv \mathrm{CH}(120 \mathrm{mg}, 1.22 \mathrm{mmol})$ in dry THF $(4 \mathrm{~mL})$ stirred at $-78^{\circ} \mathrm{C}$ under argon. The mixture was stirred at this temperature for $5 \mathrm{~min}$ and then at ambient temperature for another 10 min. A THF solution of dried $\mathrm{ZnBr}_{2}(1.3 \mathrm{~mL}, 1.0 \mathrm{M}, 1.30 \mathrm{mmol})$ was introduced via a syringe. The mixture was stirred at ambient temperature for $5 \mathrm{~min}$ (to afford a $0.2 \mathrm{M}$ solution of the zincated acetylene), before transferred to a flask containing $\mathrm{Pd}\left(\mathrm{PPh}_{3}\right)_{4}(20 \mathrm{mg}, 0.017 \mathrm{mmol})$ stirred at $-78^{\circ} \mathrm{C}$ under argon. A solution of the bromoallene $(74 \mathrm{mg}$, $0.34 \mathrm{mmol})$ in dry THF $(2 \mathrm{~mL})$ was then introduced. After completion of the addition, the mixture was stirred at $0^{\circ} \mathrm{C}$ until TLC showed disappearance of the starting bromoallene (ca. $5 \mathrm{~min}$ ). The mixture was diluted with $\mathrm{Et}_{2} \mathrm{O}$, washed in turn with water and brine, and dried over anhydrous $\mathrm{Na}_{2} \mathrm{SO}_{4}$. Removal of the solvent and column chromatography (30:1 PE/Et ${ }_{2} \mathrm{O}$ ) gave the coupling product 15 as a colorless oil (70 mg, $0.27 \mathrm{mmol}, 80 \%$ yield): $[\alpha]_{\mathrm{D}}{ }^{14}+113.7$ (c 0.85 , $\left.\mathrm{CHCl}_{3}\right) .{ }^{1} \mathrm{H}$ NMR $\left(300 \mathrm{MHz}, \mathrm{CDCl}_{3}\right) \delta 5.58-5.50(\mathrm{~m}, 2 \mathrm{H}), 4.66(\mathrm{dq}, J=2.1,6.5 \mathrm{~Hz}, 1 \mathrm{H}), 4.17(\mathrm{dd}, J=8.3,6.1 \mathrm{~Hz}$, 1H), 3.79 (dd, $J=8.4,6.4 \mathrm{~Hz}, 1 \mathrm{H}$ ), 1.46 (s, 3H), 1.41 (s, 3H), 0.20 (s, 9H); FT-IR (film) 2987, 2157, 1953, $1251 \mathrm{~cm}^{-1}$; ESI-MS m/z $259.1\left([\mathrm{M}+\mathrm{Na}]^{+}\right)$; ESI-HRMS calcd. for $\mathrm{C}_{13} \mathrm{H}_{20} \mathrm{O}_{2} \mathrm{SiNa}\left([\mathrm{M}+\mathrm{Na}]^{+}\right)$259.1131; found 259.1125.

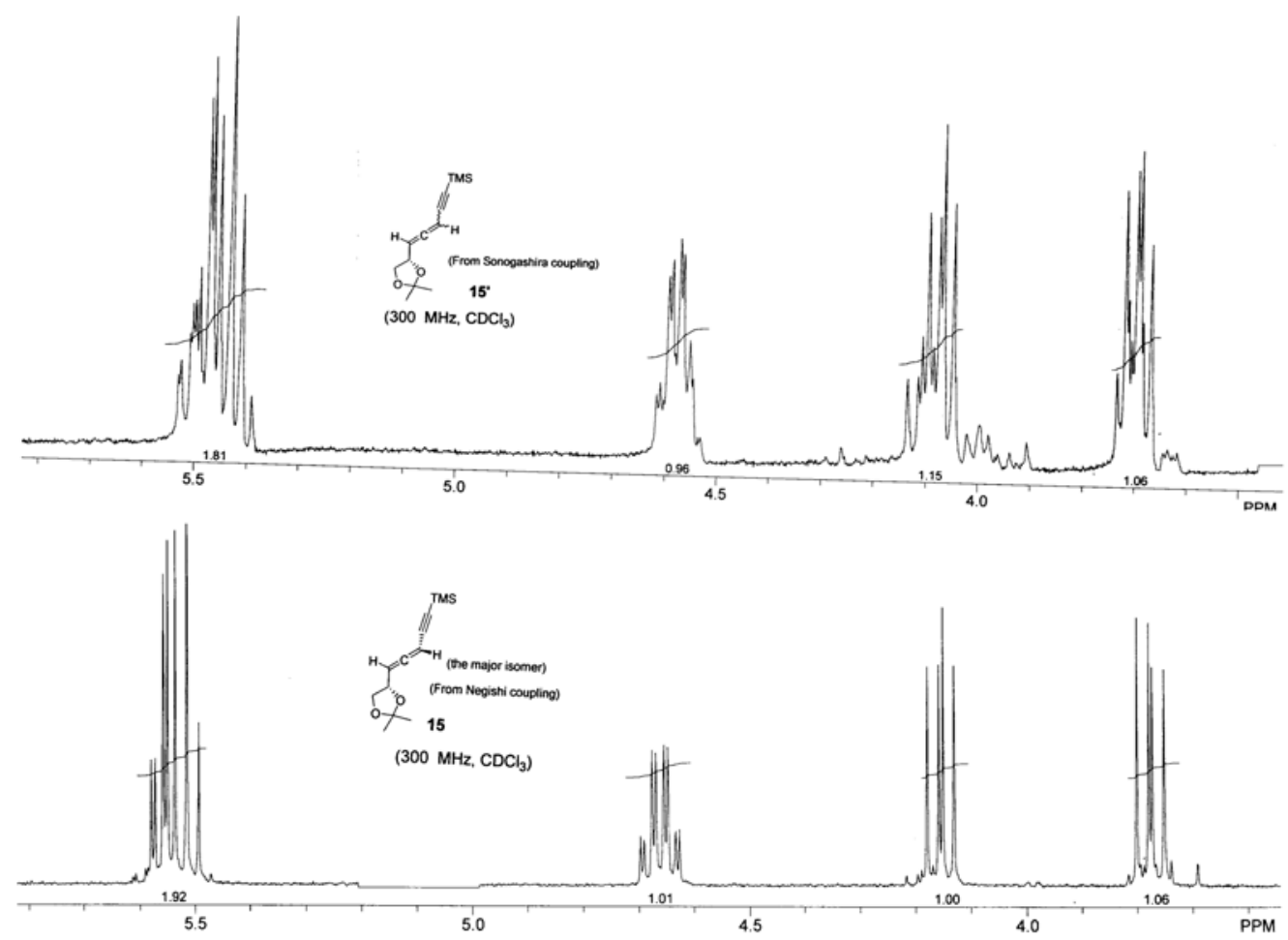

Figure S. Comparison of the partial ${ }^{1} \mathrm{H}$ NMR spectrum of $\mathbf{1 5}$ ' and 15. The coupling product under the Negishi conditions was of much better diastereomeric purity than that under the Sonogashira conditions. 


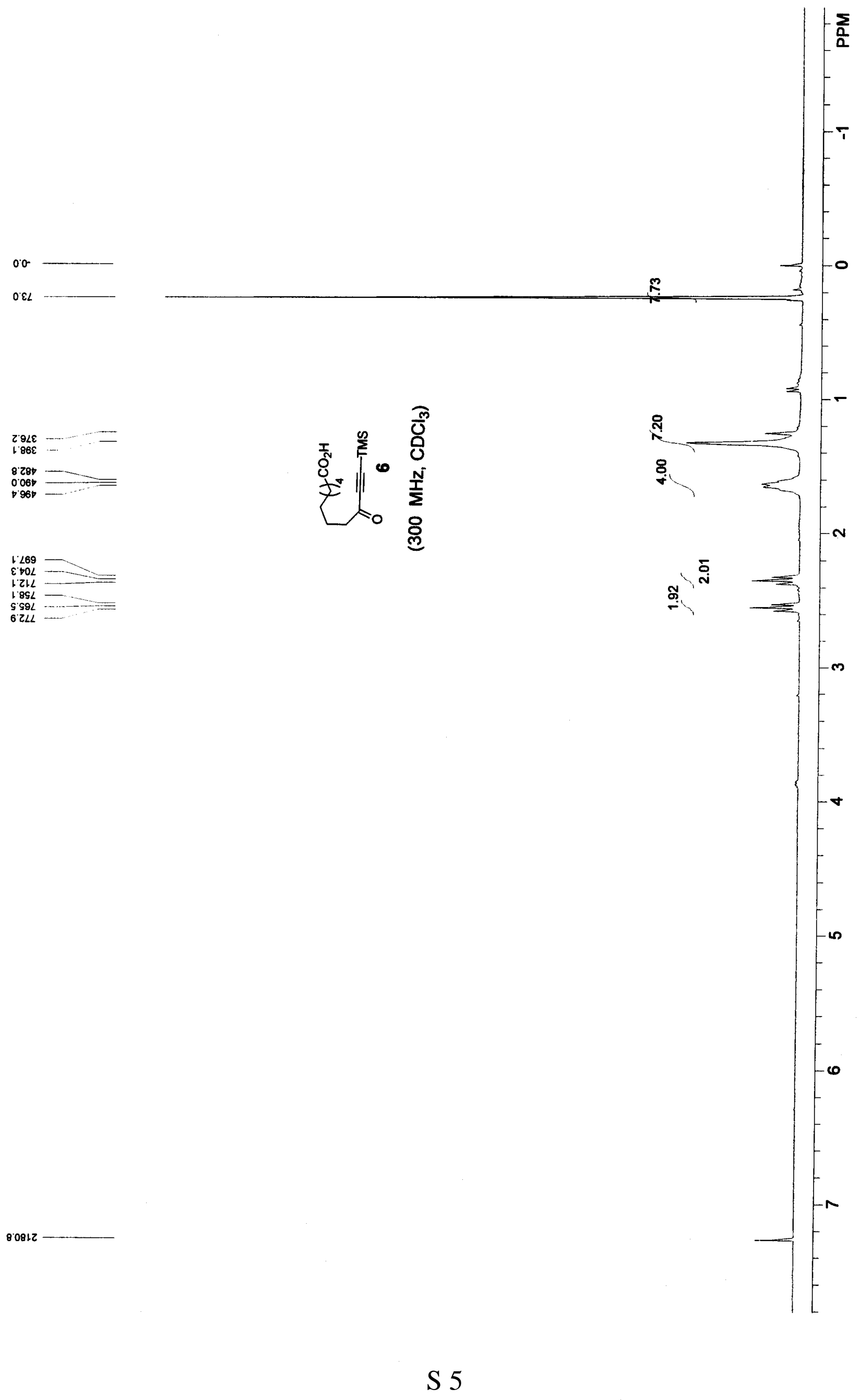




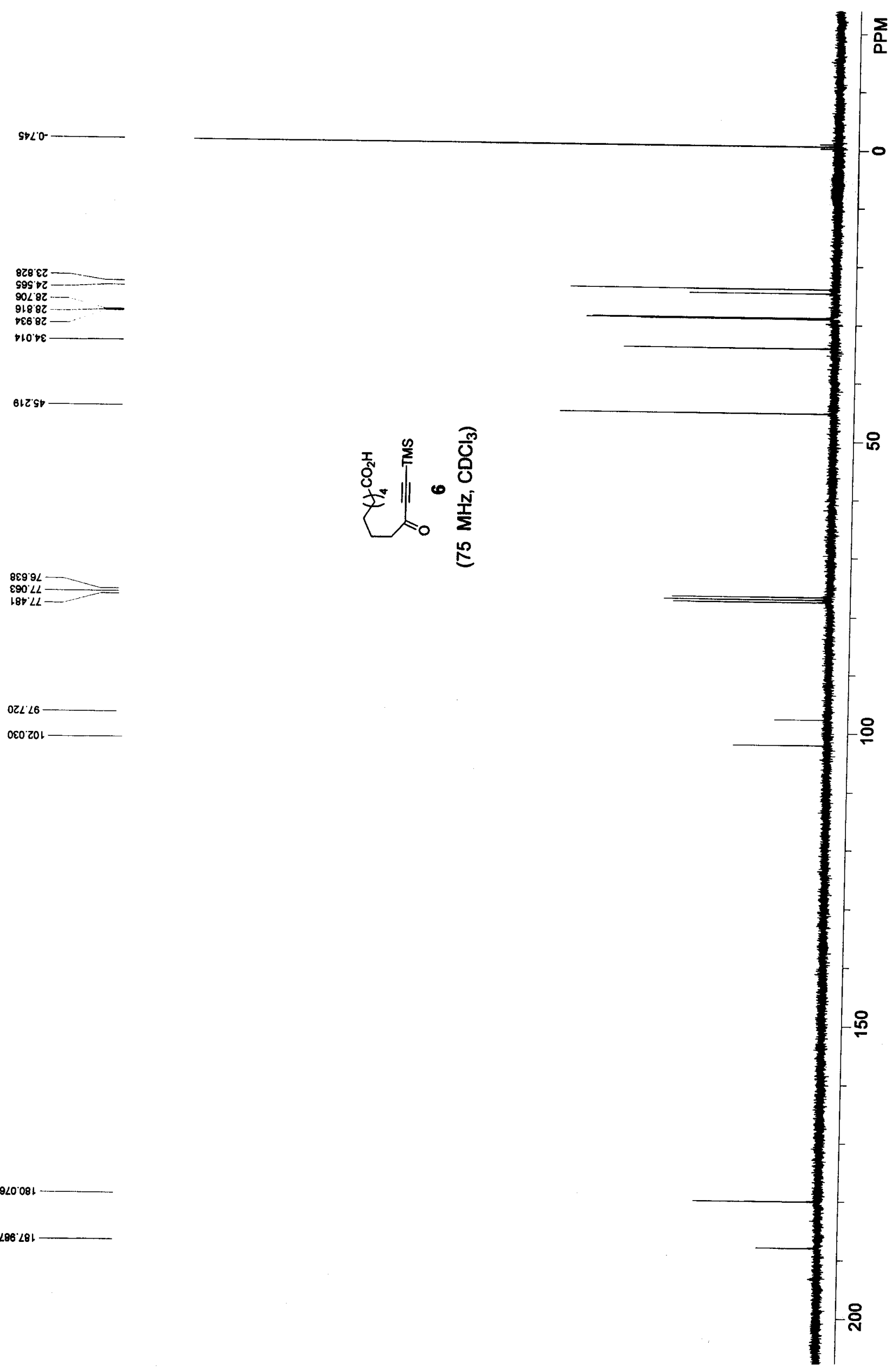

S 6 

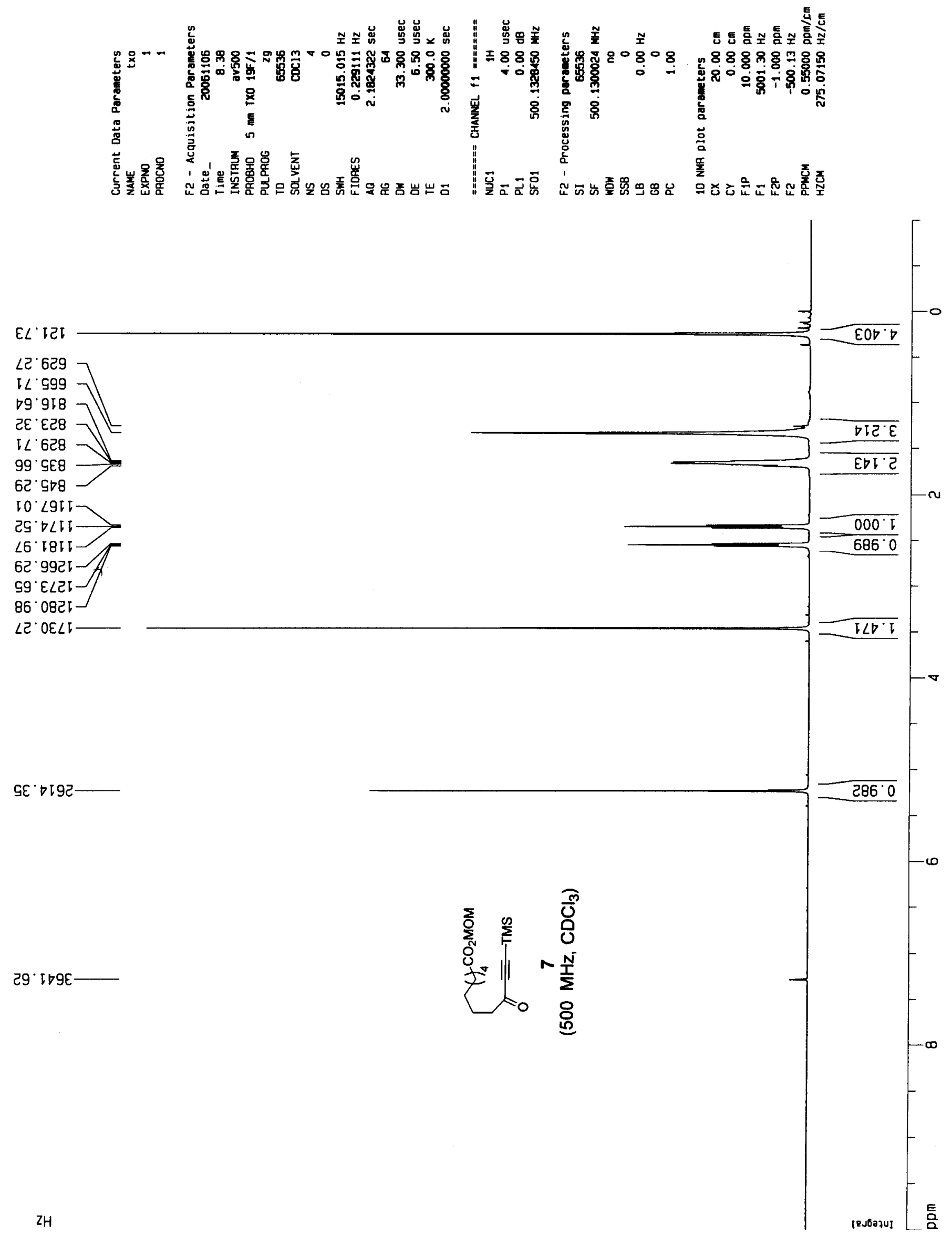
$\angle 6 L^{\circ} O-$

9LL'EZ

S99. $\square$

$\square .82-$
EI8. $85-$

ट68.85

II $\vee V E$

$2 \angle \downarrow \cdot G \nabla$

$00 S^{\circ} \angle S^{-}$

$99 L^{\prime} 9 L$

$020^{\circ} \angle L$

$D \angle C^{\prime} \angle L$

$2 \angle 5 \cdot 06$

OSG ' $\angle 6$

980.201

$90 Z^{\circ} \varepsilon \angle \digamma-$

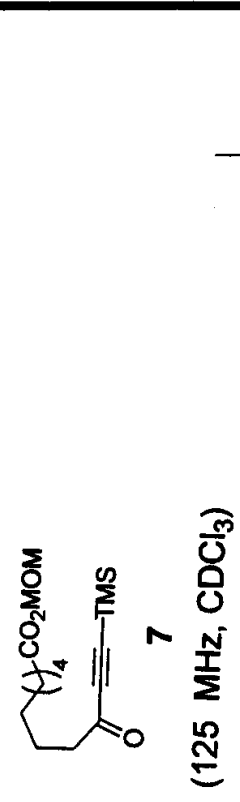

$66 L^{\circ} \angle 8 I$

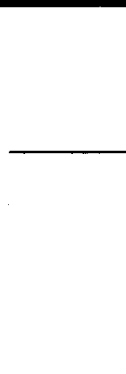




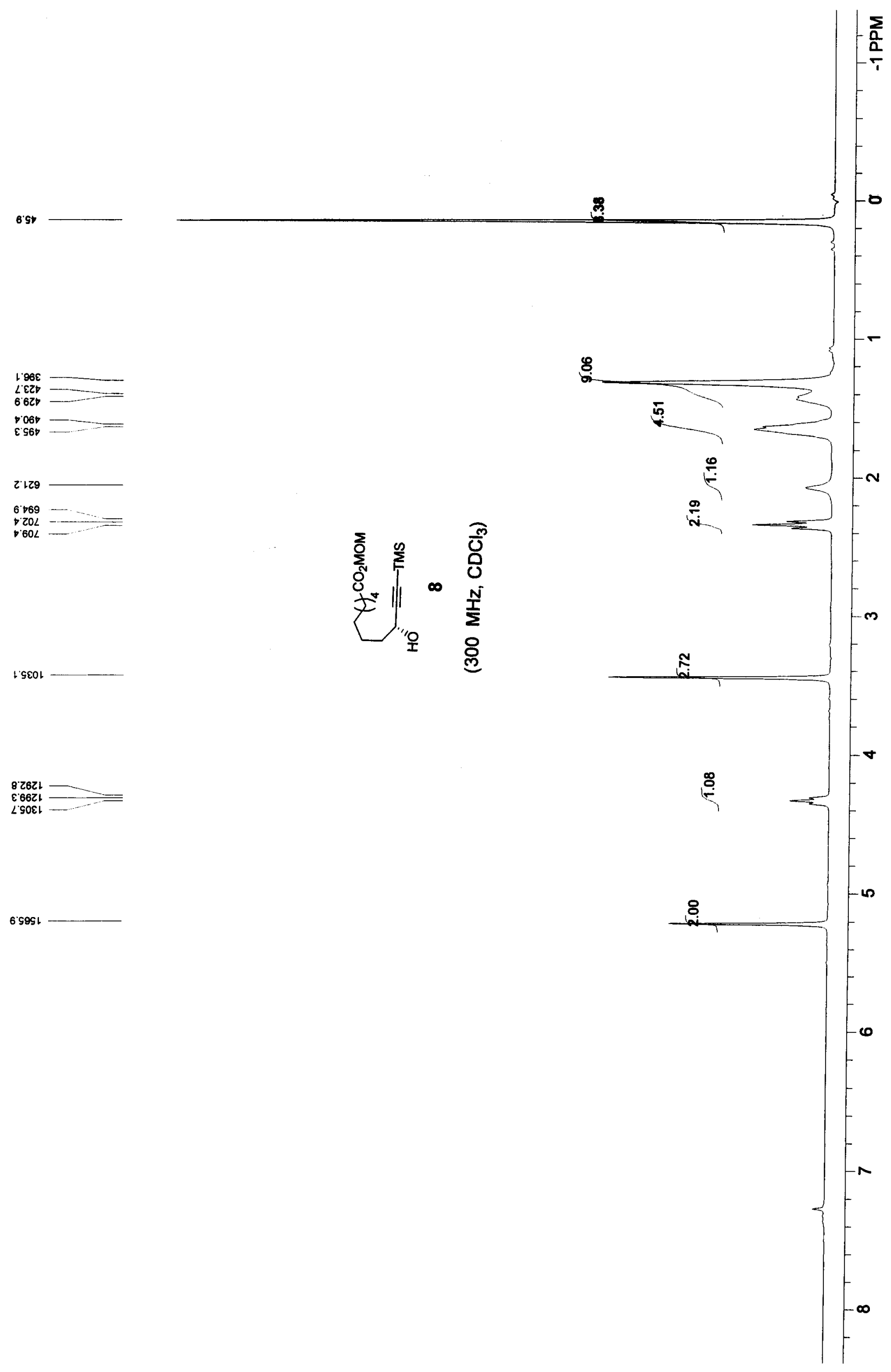


LIS LE

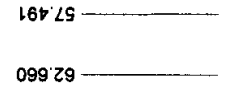

$995 \cdot 92$

$786.9 L$
GLt $L L$

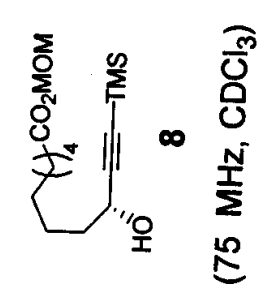

$\angle 8068$

28006

898.901

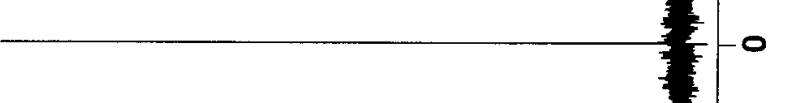

$\frac{10}{80}$ 

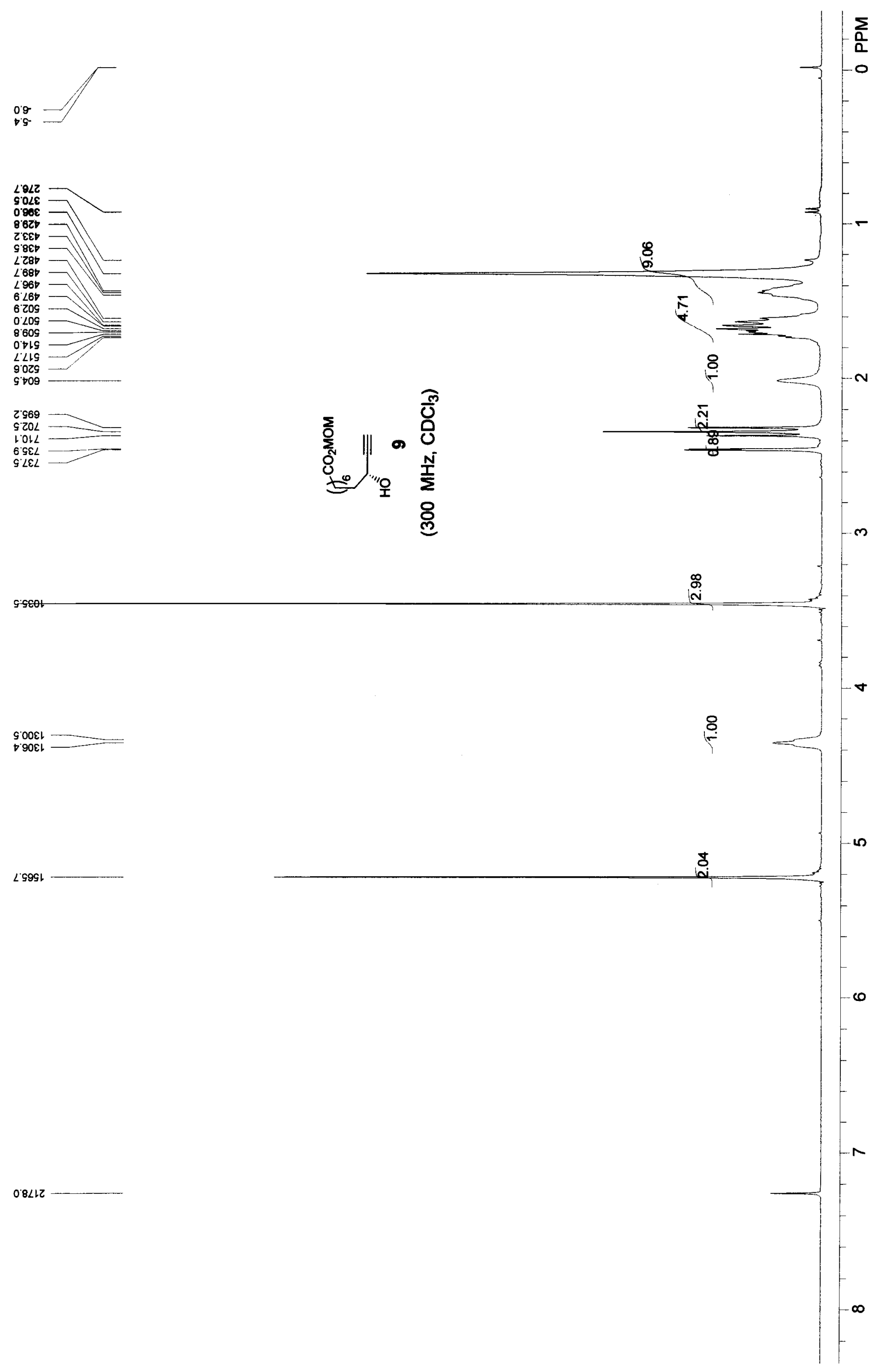

g.008

S 11 

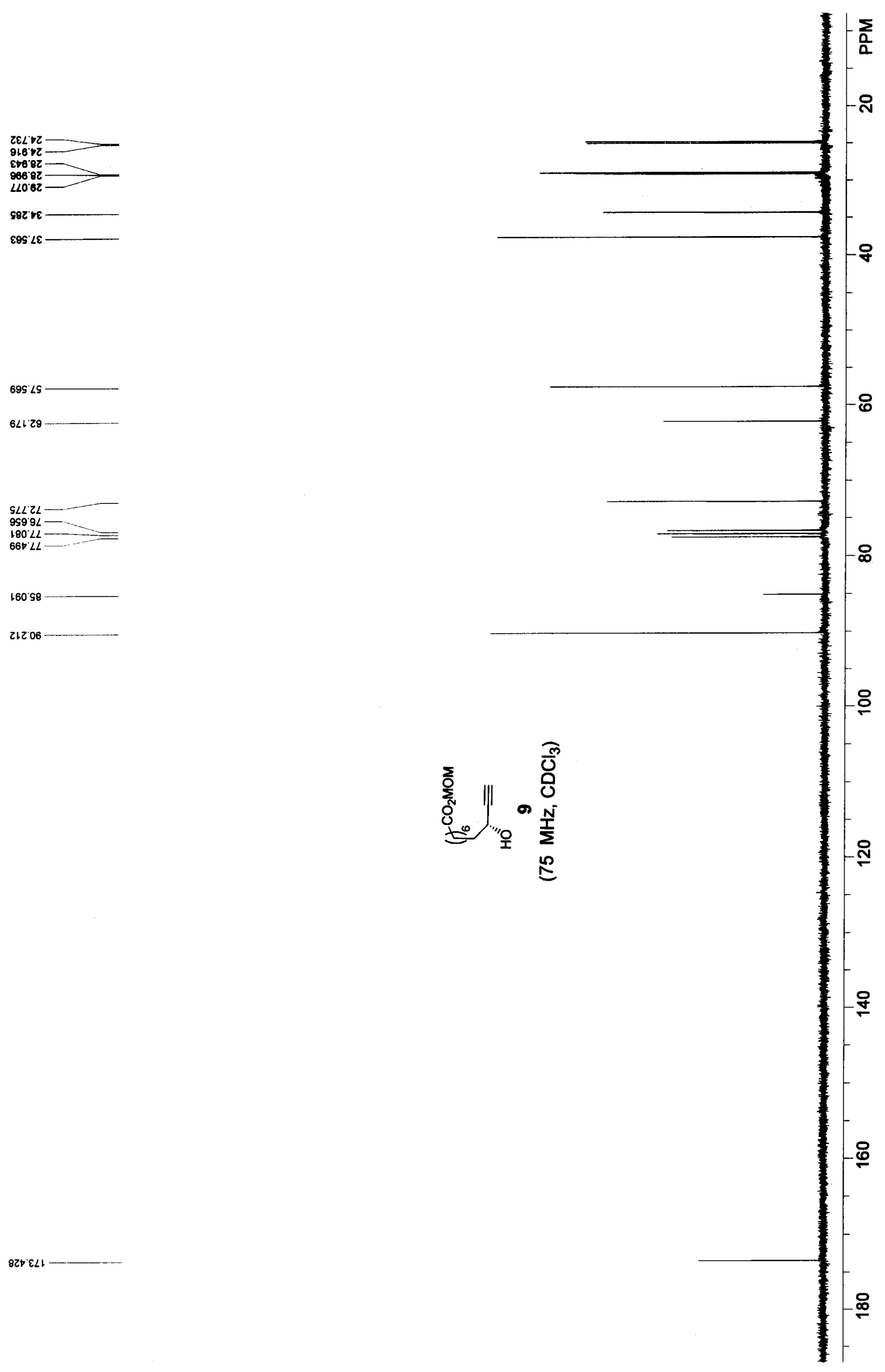

$8 z+\varepsilon<1$ 

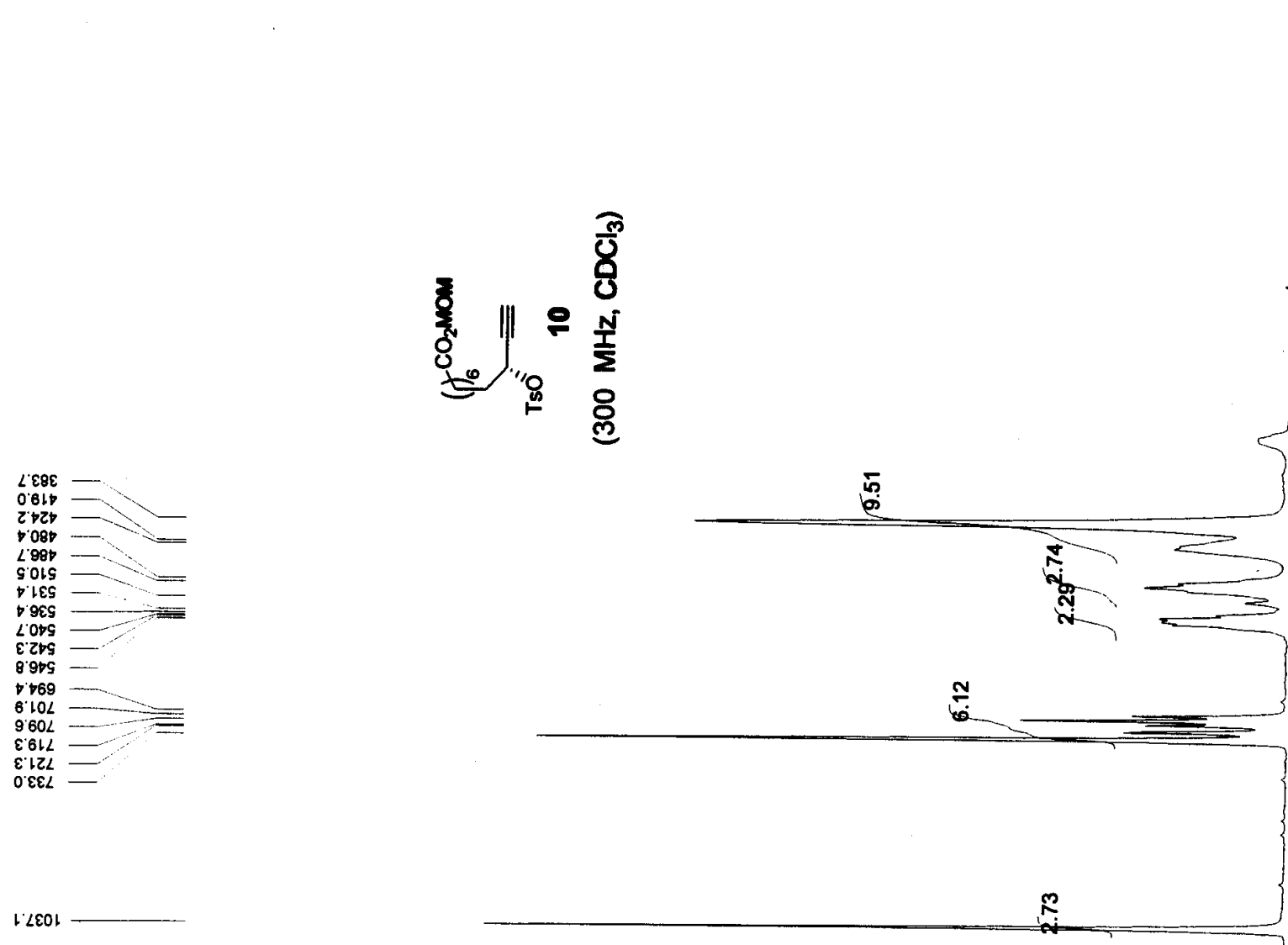

r $<\varepsilon 0 เ$

||$\frac{z}{0}$

Lit 9095

Zilst

LLLSL.

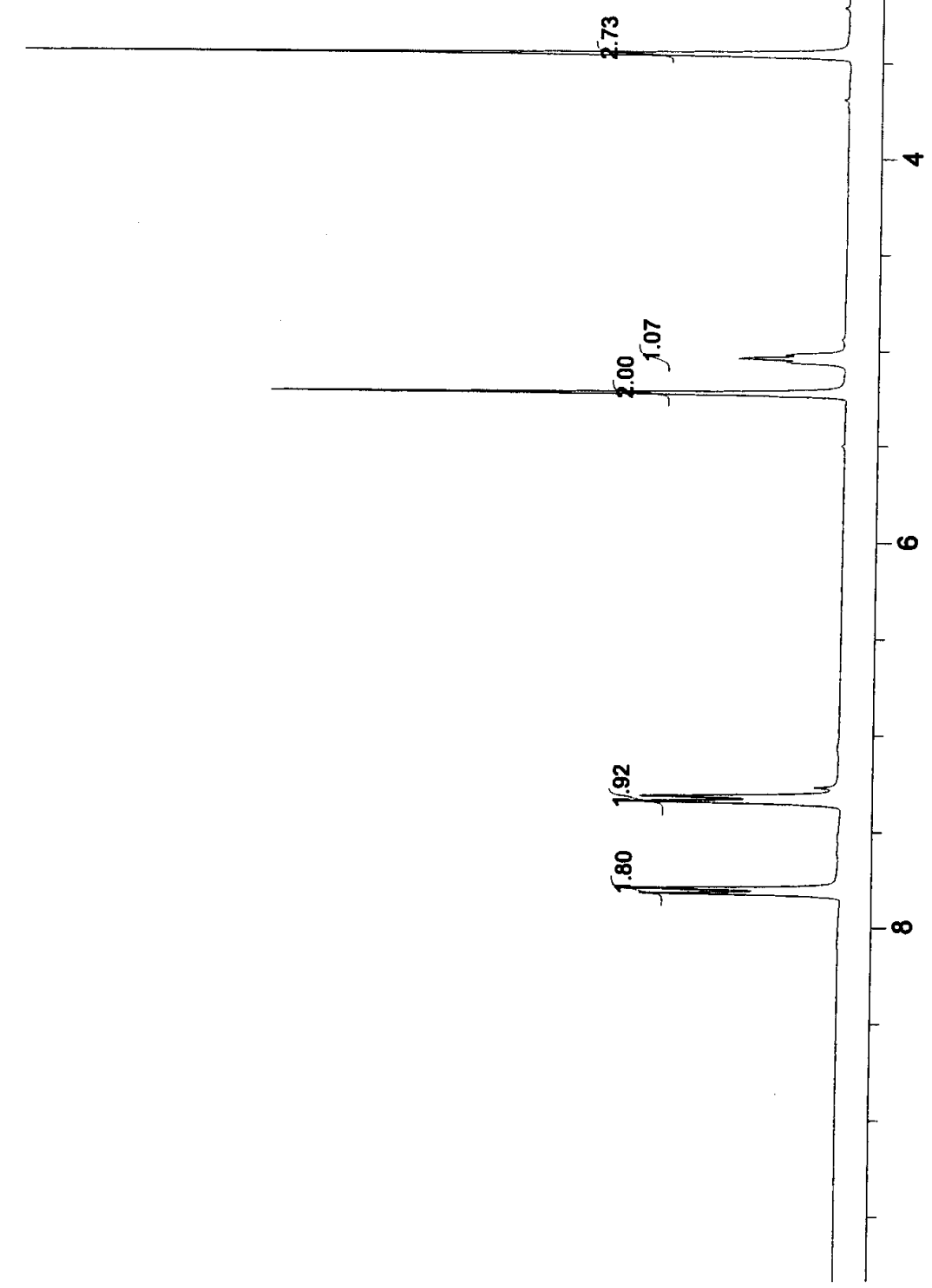

$099612-\cdots$
8
8
-

乙'в६६z -

8. 


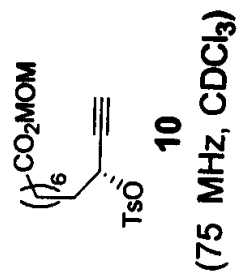

$68+29$

$286^{\prime} 0 L$

$960.9 L$

$686.9 L$

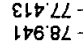

280.06

SGL $\downarrow \varepsilon$$$
\text { เDE } 82
$$

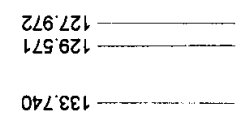

O† $\angle \varepsilon E L$

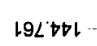

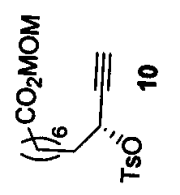

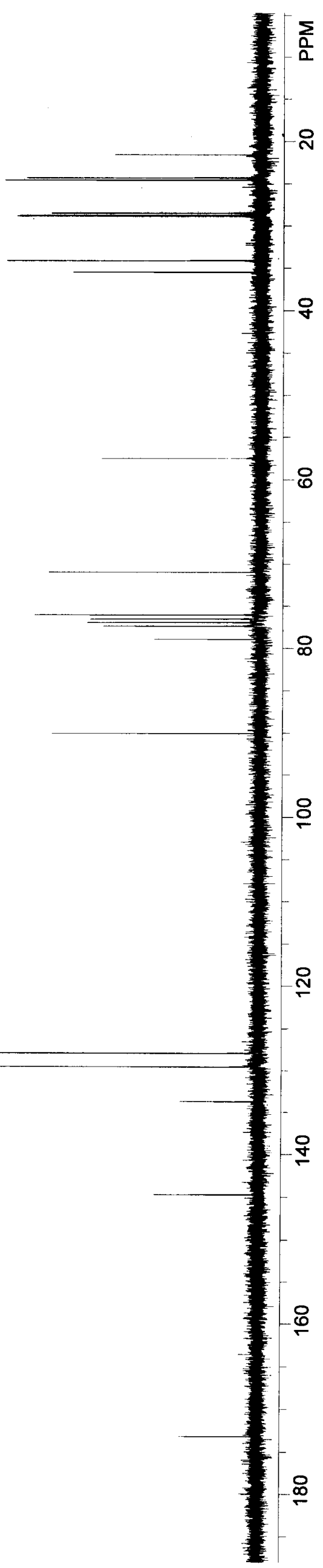

오

LOZ\& $\angle L$ 
Eเ-

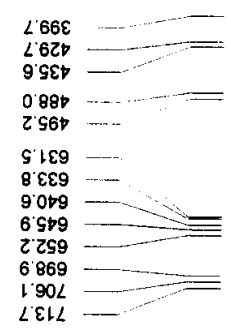

s.88ol

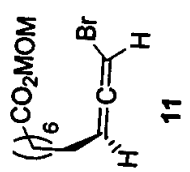

16

\&.8091
l. 191

lol9t - -

80291

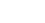

$\varepsilon 8 L L b-\ldots$
$208 L b-\ldots$

LOB LL -

(1)

02812

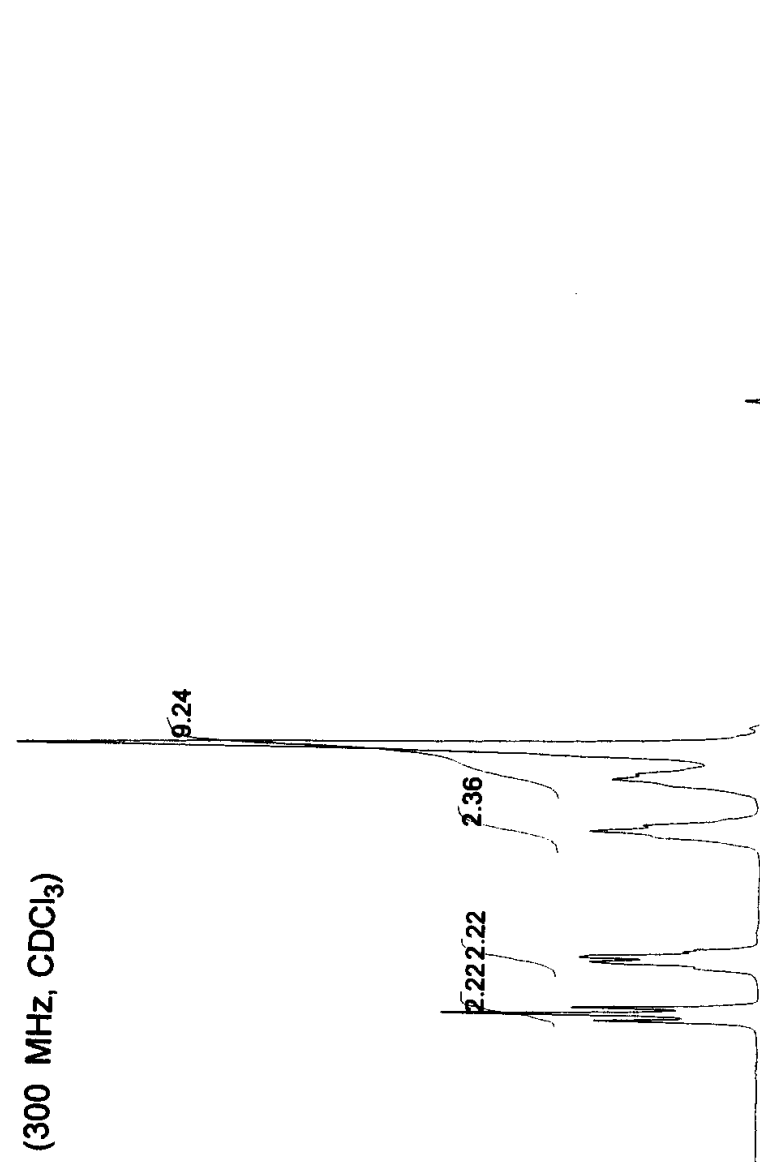

高

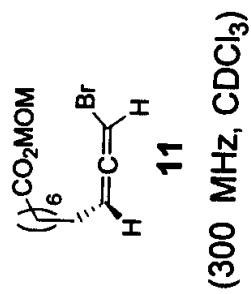

$\stackrel{\infty}{\circ}$
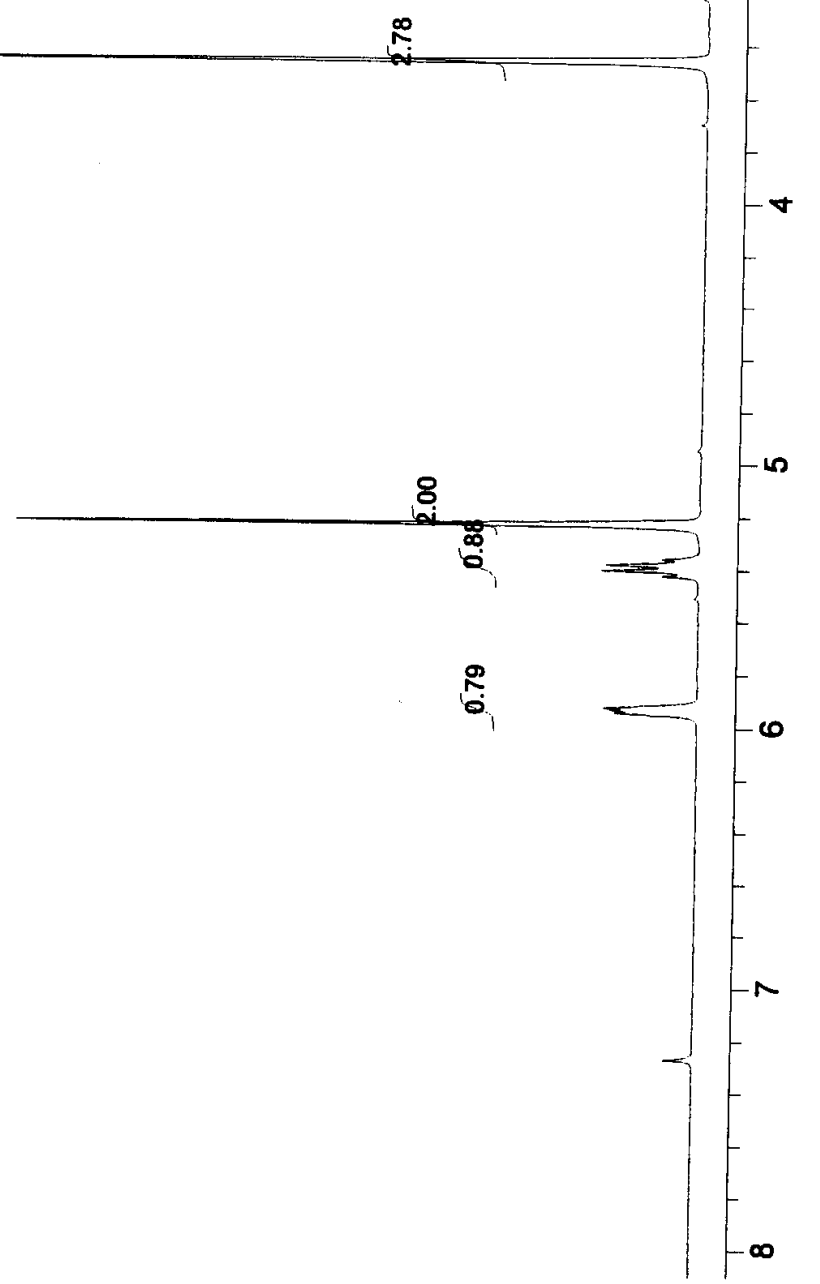

S 15 

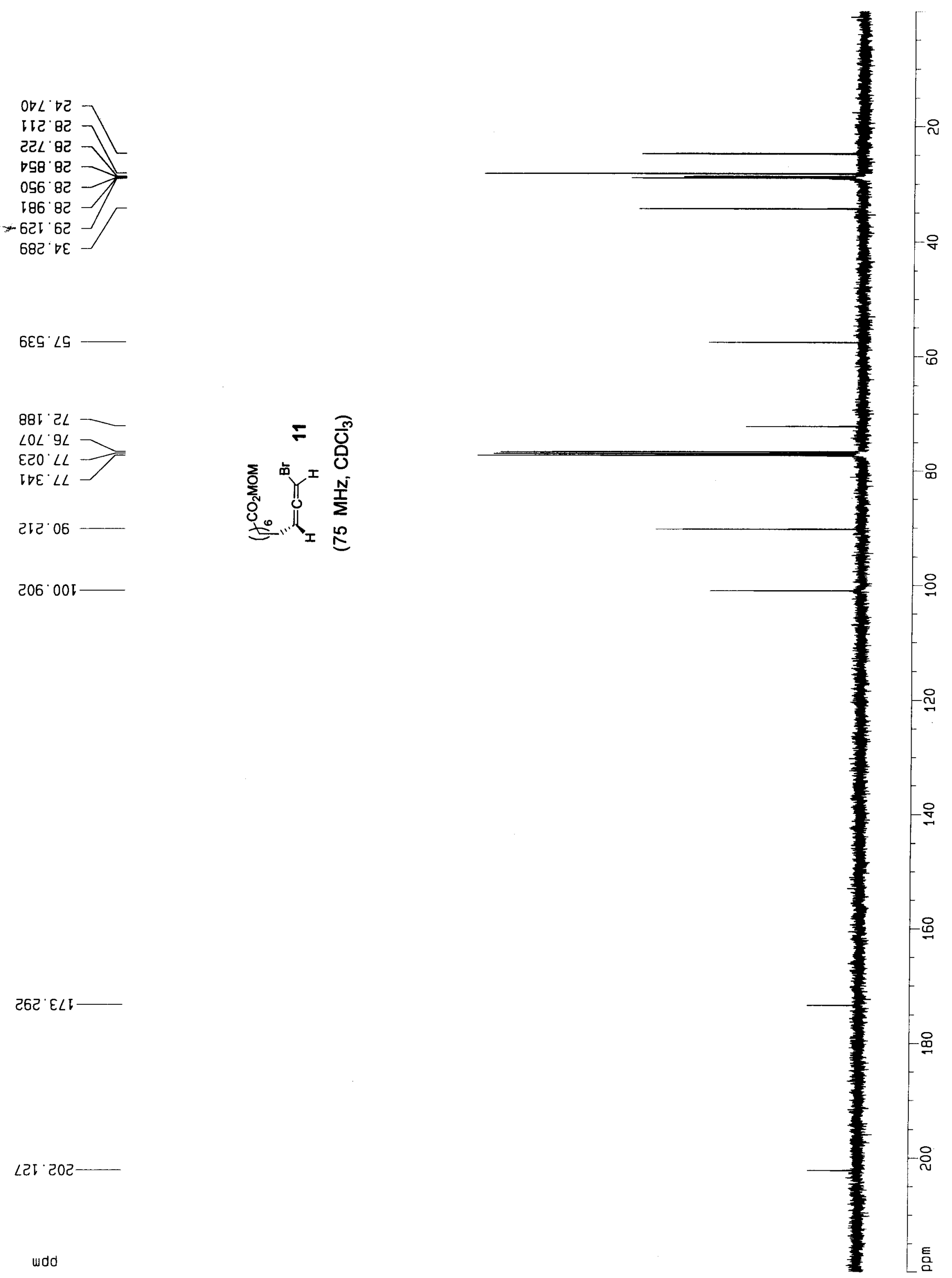

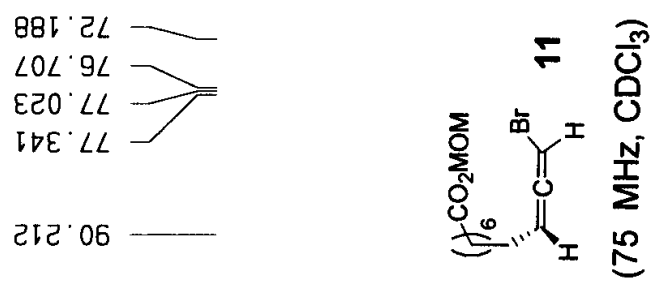

$206 \cdot 005$

$26 \mathcal{C}^{\prime} \varepsilon \angle I-$

$\angle 2 I 202$ 


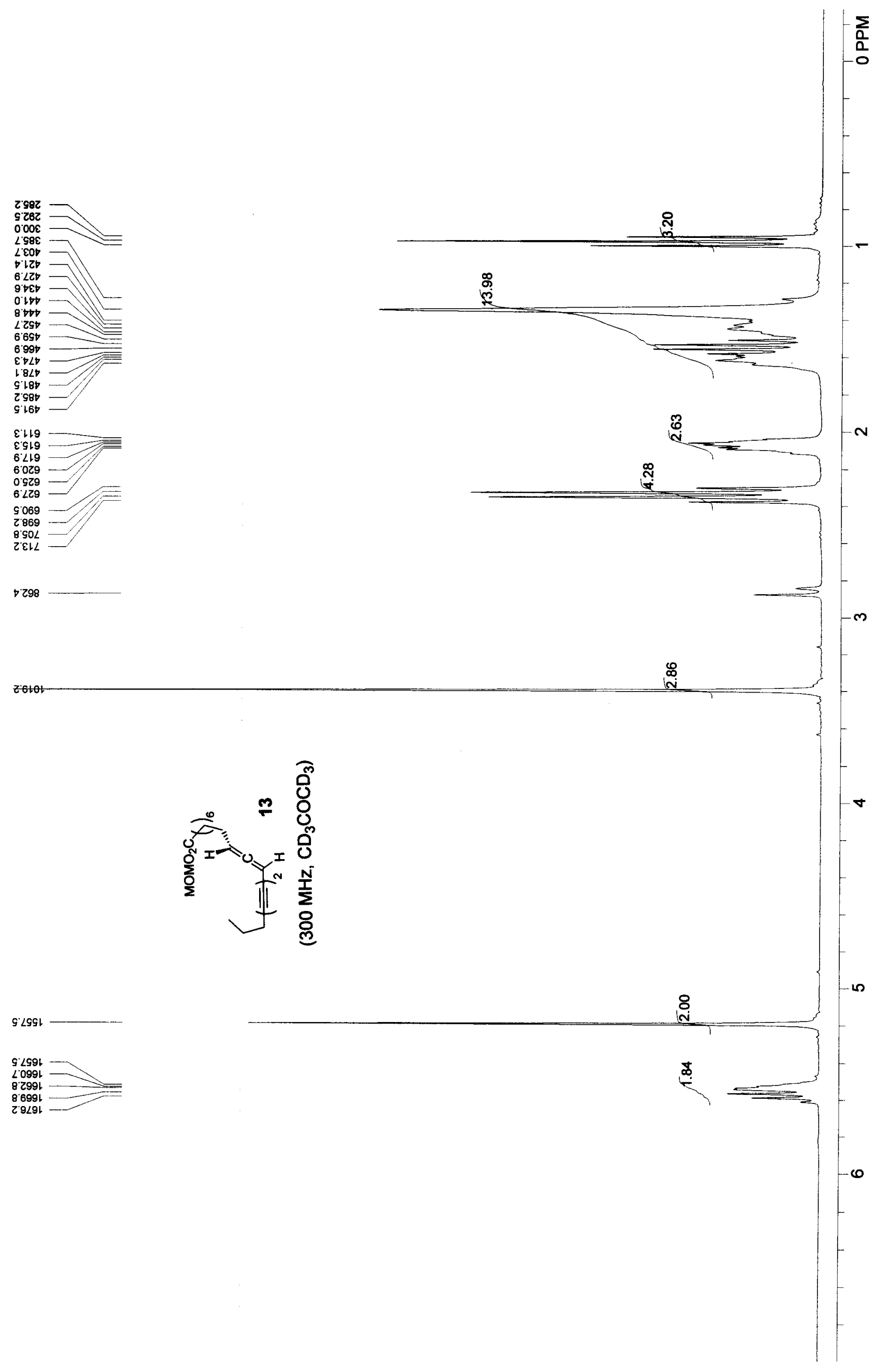




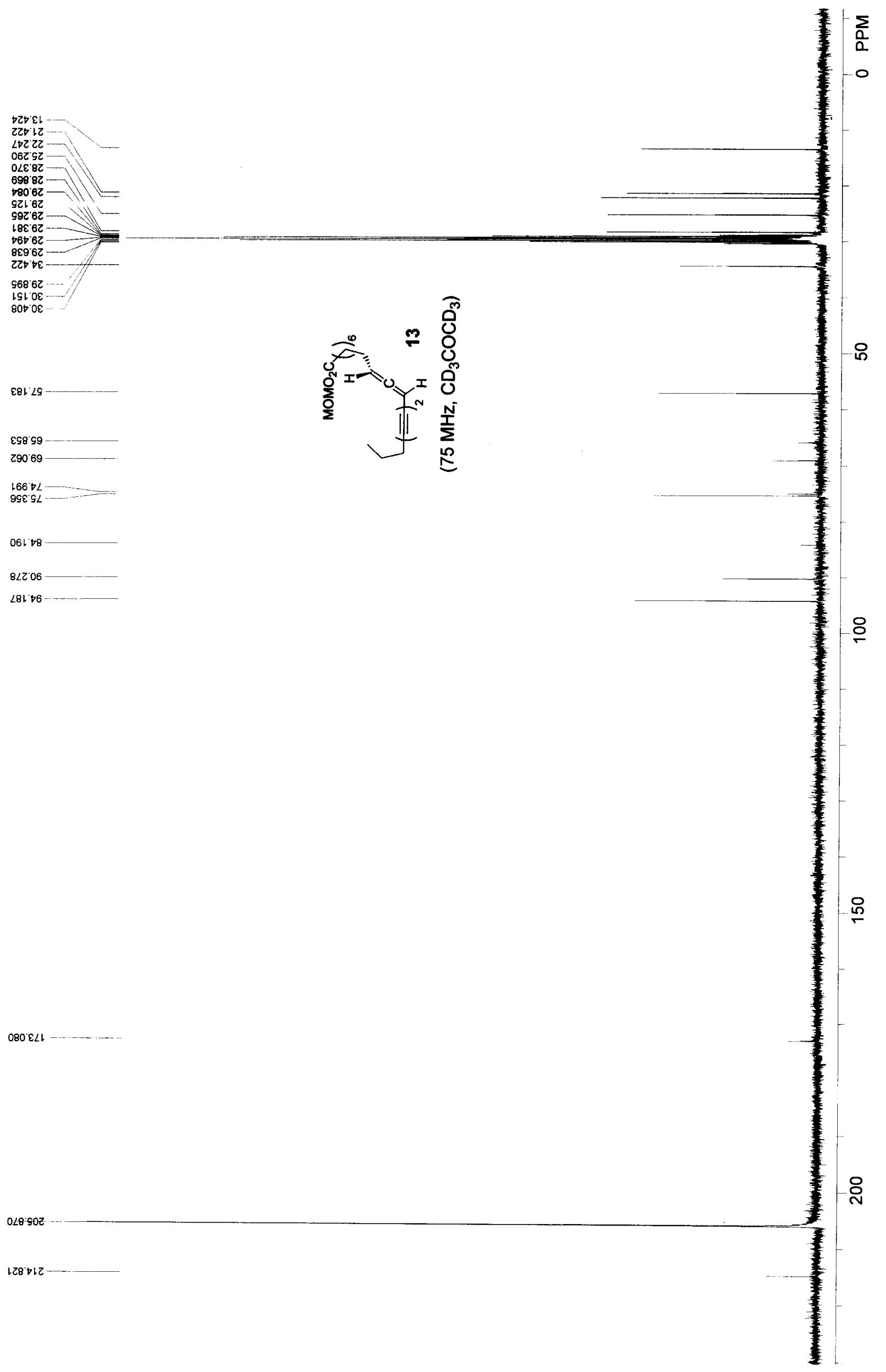



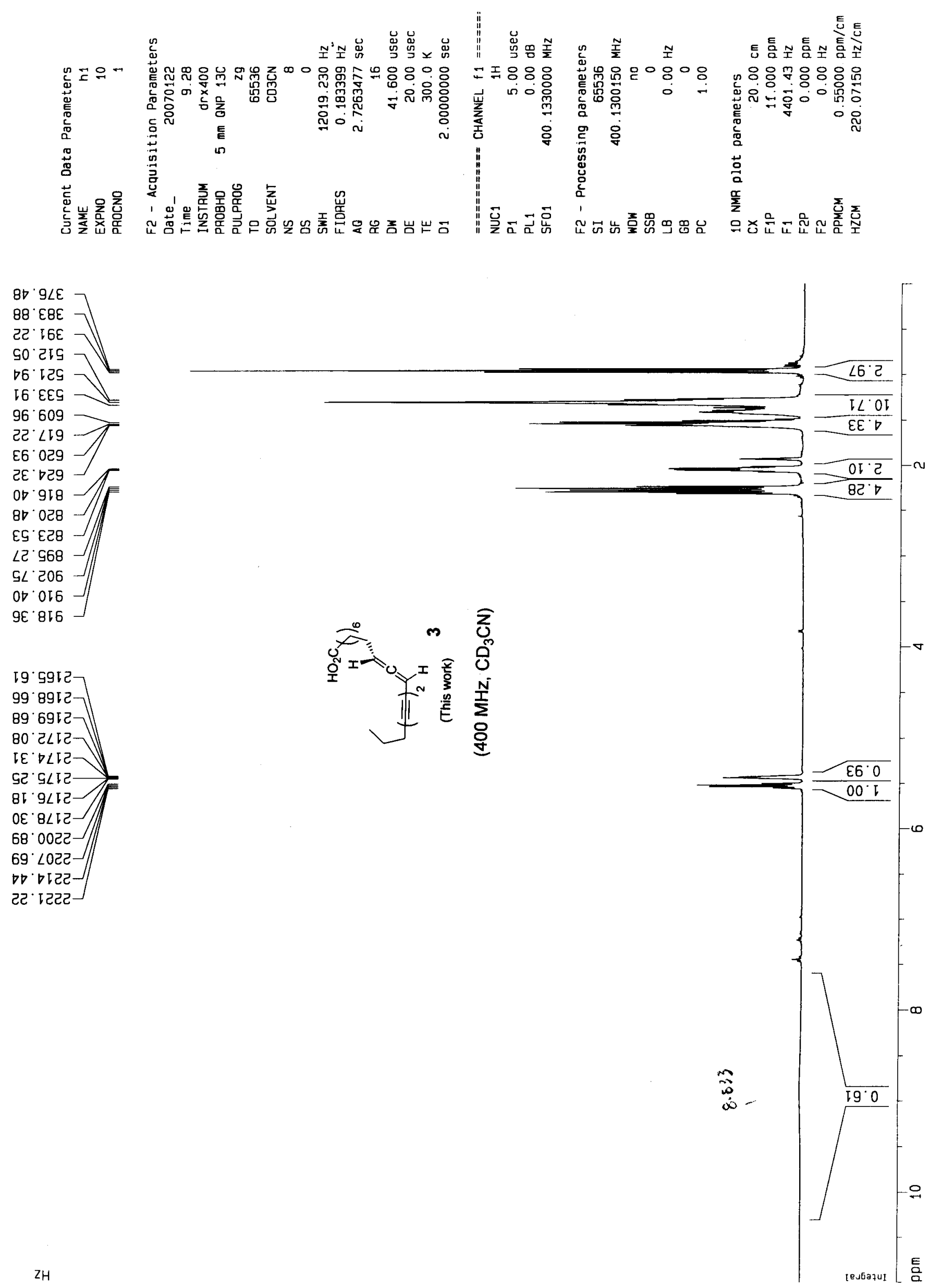


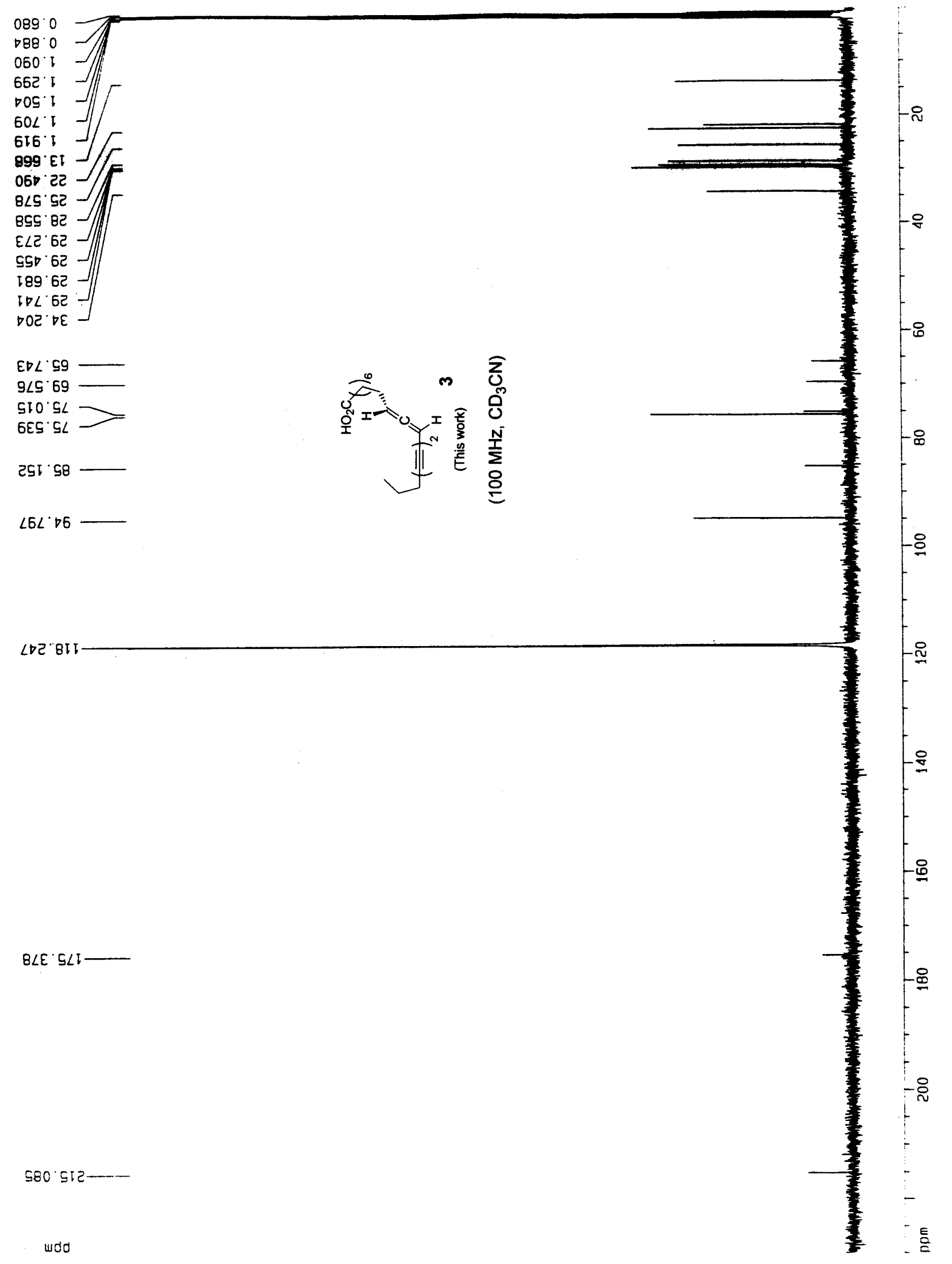


เZE।

$68 \varepsilon L 1$
$01+1 L$

L $\angle D L L$

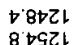

8 tgCL

6 Z9ZL

$\angle 968$ L

$6 \angle 6 \varepsilon l$
E $D O D L$
$\angle O L b L$

LOLt

9ltb

$8<10$

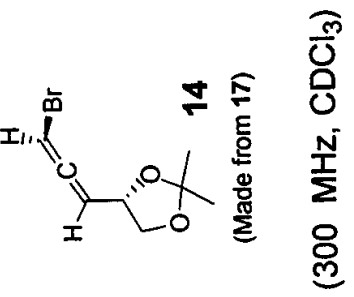

เ. 291

ร LZ91

9 เع 
2801

rort

$t$ ZL

$1+t$

9ozl

$\varepsilon \varepsilon$

GStz

9.12

$8 \varepsilon Z Z$
$\angle 9 Z Z$

6622

Gizez

เ 'รEZ

t'998

b $S \angle \varepsilon$

$69 \angle \varepsilon$

$\forall \forall 9 l$
$\angle 0 \varepsilon 91$

$S<\varepsilon 9$

$9+5:$

$\angle$ Ero

los9

6059

0.7cor

ع
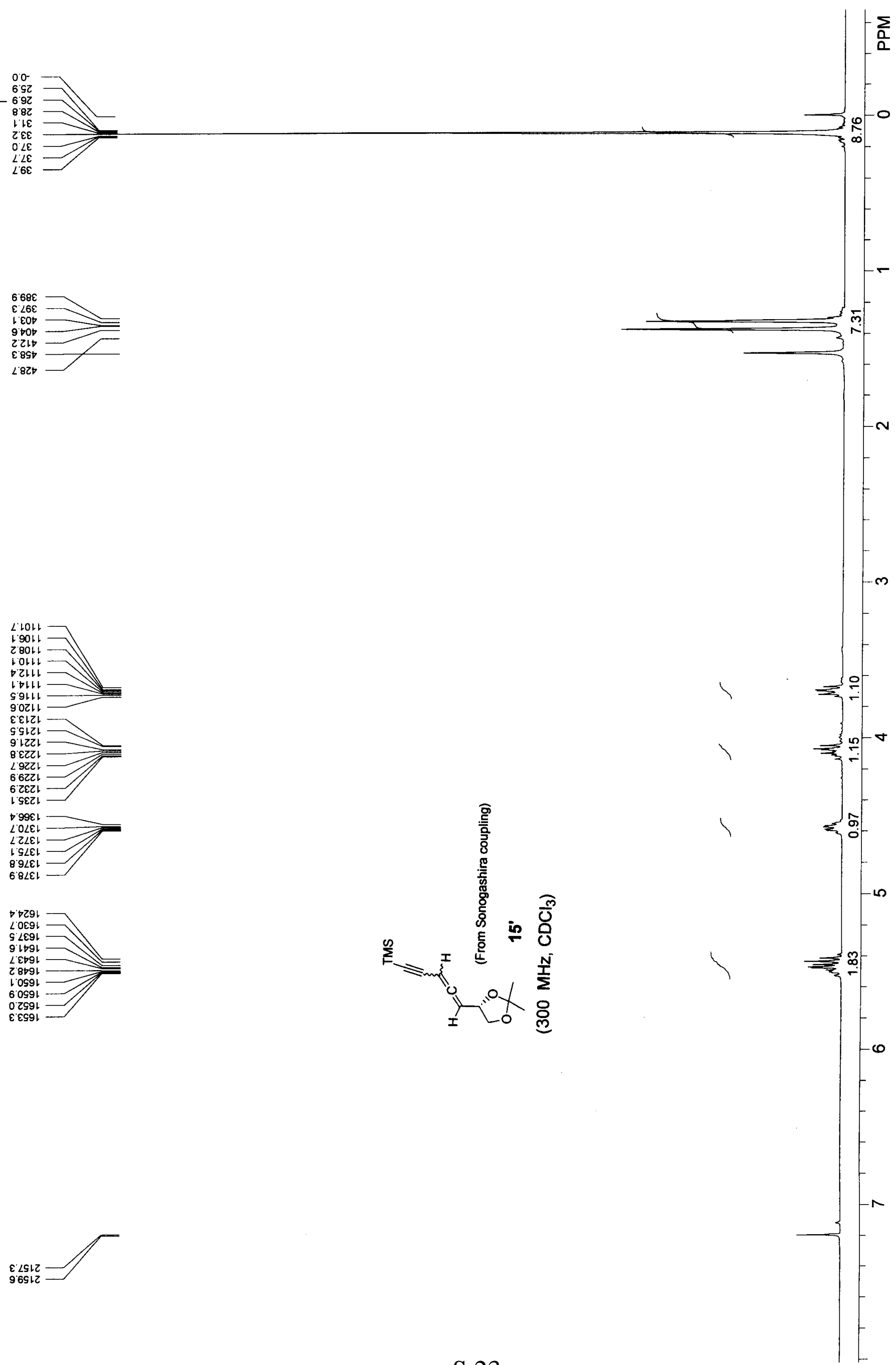


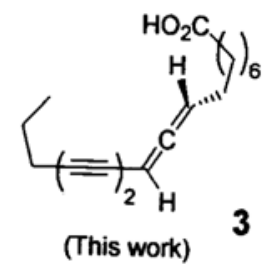

( $400 \mathrm{MHz}, \mathrm{CD}_{3} \mathrm{CN}$ )
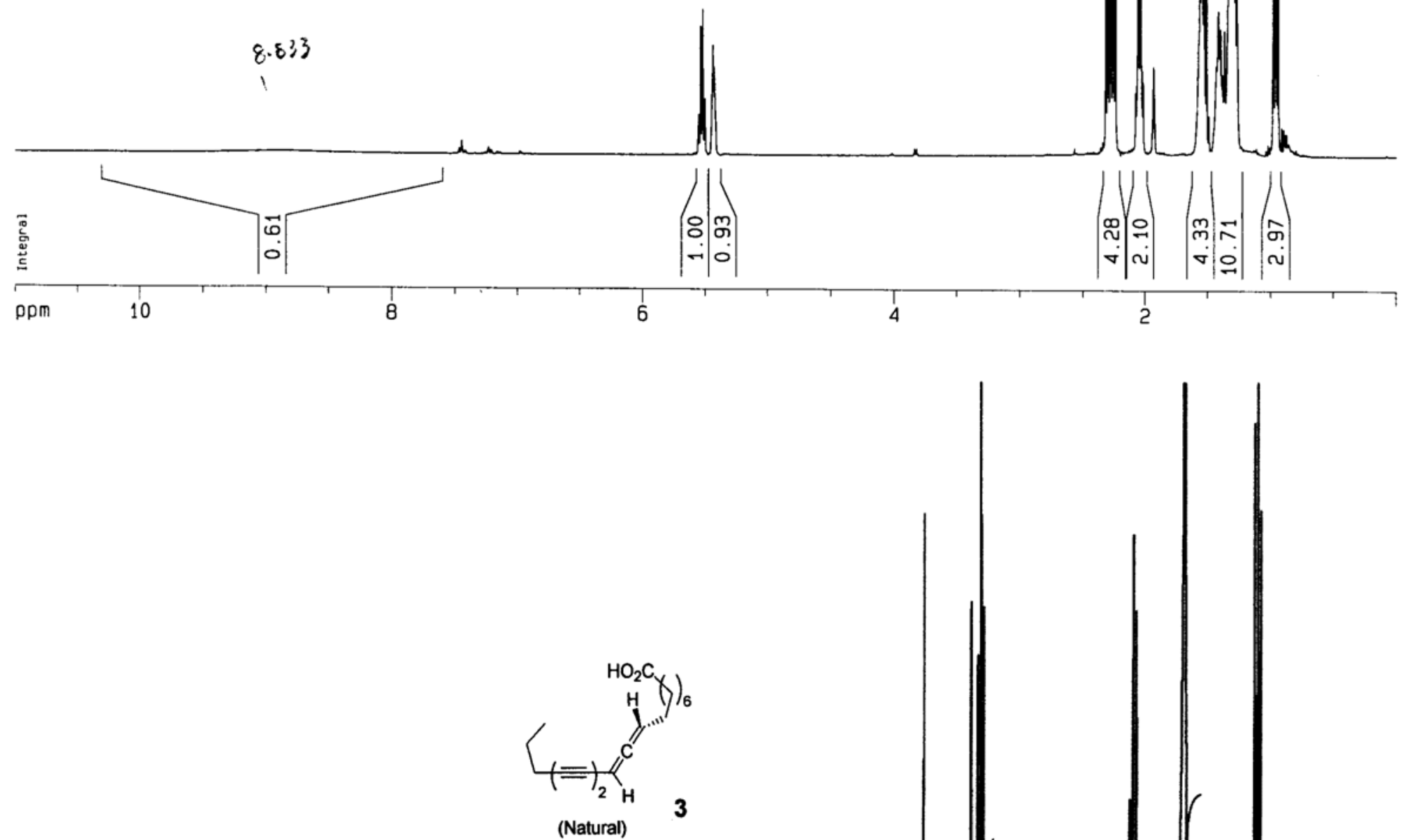

Copied from ref $1 a$

(500 MHz, $\mathrm{CD}_{3} \mathrm{CN}$ )

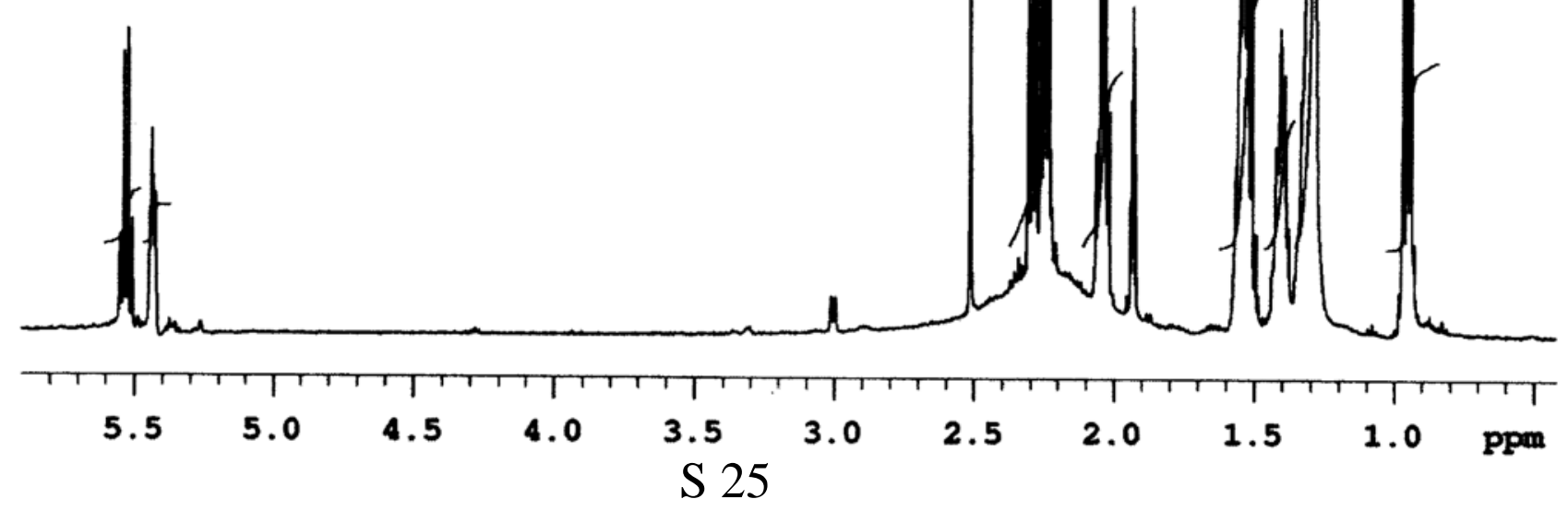


言

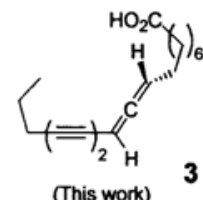

(100 MHz, $\mathrm{CD}_{3} \mathrm{CN}$ )
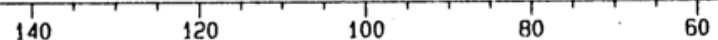

20

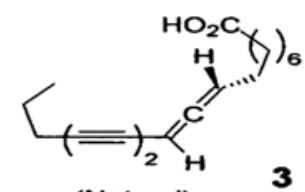

(Natural)

Copied from ref $1 a$

(125 $\mathrm{MHz}, \mathrm{CD}_{3} \mathrm{CN}$ )

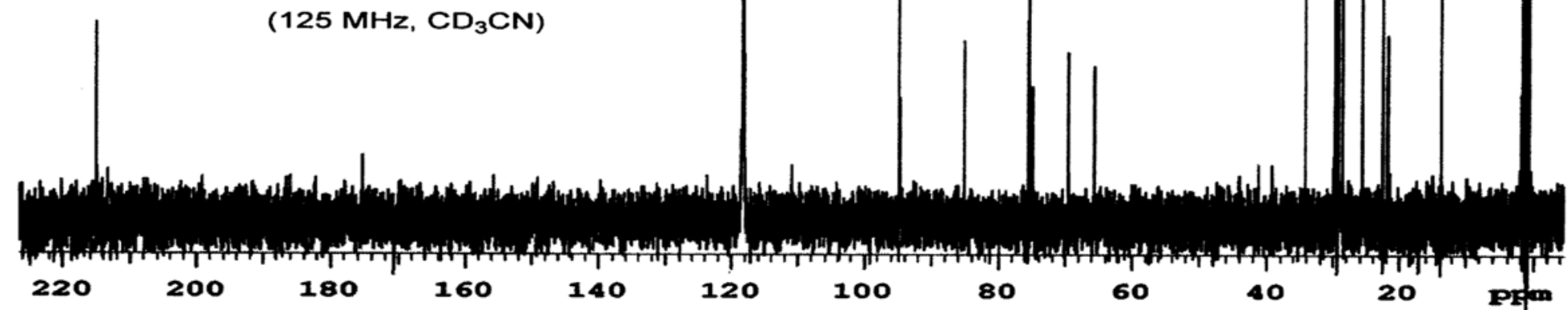




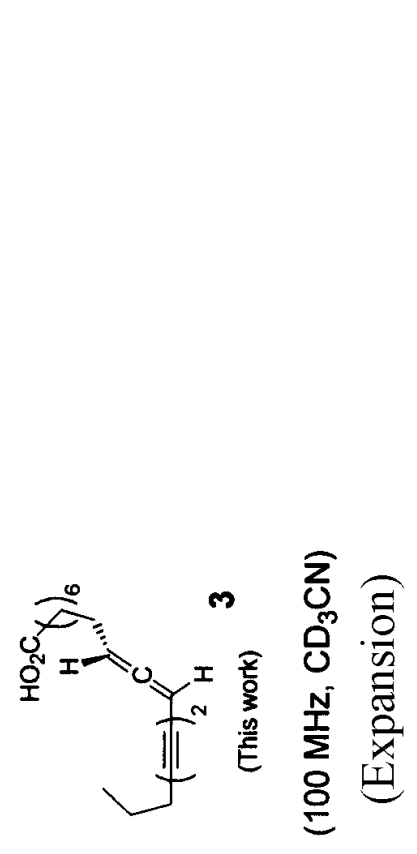

$6 \angle L S^{\circ} g 己$

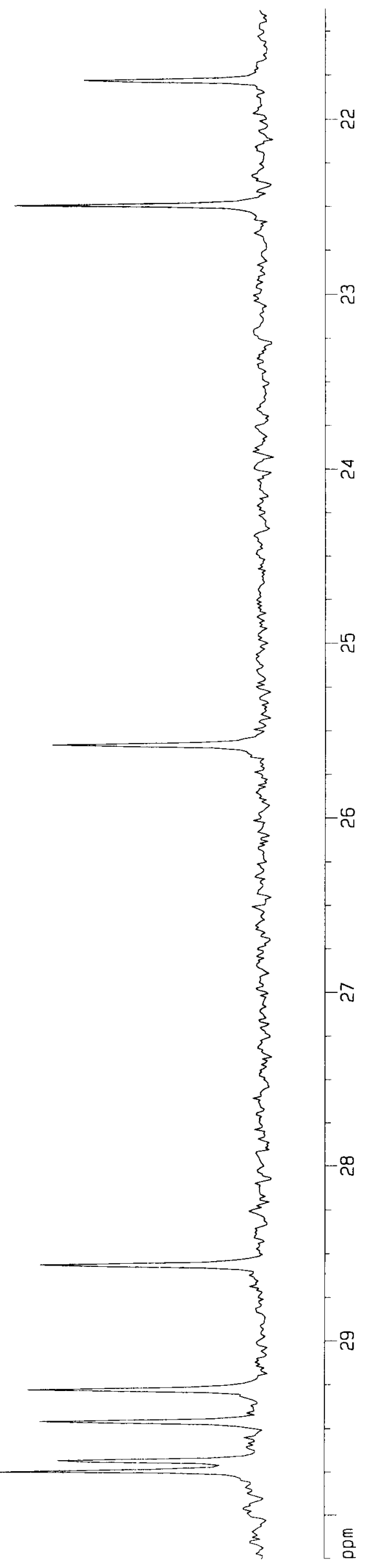

OEL己 $6 己$

टGSD 62

$8089^{\circ} 6 \mathrm{C}$

I $\backslash \backslash L^{\circ} 62$

wdd 